\title{
EVALUACIÓN DE LA EXPOSICIÓN OCUPACIONAL A RUIDO EN MICROEMPRESAS DE MADERA DE LA CIUDAD DE NEIVA EN EL 2019
}

\section{OCCUPATIONAL NOISE EXPOSURE ASSESSMENT IN WOODEN MICROENTERPRISES OF NEIVA CITY IN 2019}

\section{${ }^{1}$ Ingrith M. Romero Méndez, ${ }^{2}$ Dinory Serrato Rojas, ${ }^{3}$ Roberson Daniel Bernal Medina, 4 Jhonathan Cabrera Urriago}

\footnotetext{
1Magíster en Tecnología Ambiental y Recursos Hídricos, Docente de Administración en Seguridad y Salud en el Trabajo de la Corporación Universitaria Minuto de Dios- UNIMINUTO, Neiva, Colombia.

2Magíster en Educación, Especialista en Higiene Industrial, Docente de Administración en Seguridad y Salud en el Trabajo de la Corporación Universitaria Minuto de Dios- UNIMINUTO, Neiva, Colombia.

${ }^{3,4}$ Profesional en Administración en Salud Ocupacional, Corporación Universitaria Minuto de DiosUNIMINUTO, Neiva, Colombia.

${ }^{1}$ iromeromend@uniminuto.edu.co, ${ }^{2}$ dinory.serrato@uniminuto.edu.co, ${ }^{3}$ rbernalmed1@uniminuto.edu.co, ${ }^{4}$ jcabrera1@uniminuto.edu.co
}

\section{RESUMEN}

Contextualización: la evaluación de la exposición a ruido es un componente indispensable en los programas de prevención de pérdida auditiva para reconocer trabajadores vulnerables, proponer medidas de control y/o evaluar las medidas implementadas.

Vacío de conocimiento: en Colombia, hay un número limitado de trabajos que abordan la exposición a ruido y las medidas de control de este.

Propósito del estudio: la exposición ocupacional a ruido en el área operativa de dos microempresas de la ciudad de Neiva fue evaluada mediante dosimetría y sonometría. Dichas empresas se dedican a la comercialización de madera aglomerada empleando máquinas especializadas como sierras verticales y enchapadoras.

Metodología: inicialmente se realizó una caracterización de las condiciones de trabajo, luego se realizaron las mediciones de ruido siguiendo la estrategia basada en la labor conforme la ISO 9612-2010.

Resultados y conclusiones: los niveles de presión sonora equivalente $(73,0$ y 82,4 dB $(A)$ ) encontrados a partir de sonometría 
se asemejan a los reportados en talleres de carpintería y fábricas de muebles. Los resultados de la dosimetría revelaron que los operarios de la sierra vertical y el de la enchapadora se exponen a diario a niveles de ruido entre 88,50 y $89,9 \mathrm{~dB}(\mathrm{~A})$ que sobrepasan el valor límite permisible (85 dB) para un tiempo de exposición de 8 horas, como se estipula por la resolución 1792 de 1990. Sin embargo, los operarios de las microempresas de madera aglomerada evaluados no se encuentran sobreexpuestos a ruido gracias a la atenuación que brindan los protectores auditivos utilizados.

Palabras clave: contaminación acústica; higiene industrial; industria maderera; salud ocupacional.

\section{ABSTRACT}

Contextualization: The noise exposure assessment is an essential component of hearing loss prevention programs to recognize vulnerable workers, to propose control measures and / or to evaluate the measures implemented.

Knowlegdge gap: In Colombia, there is a limited number of works that address noise exposure and its assessment.
Purpose: Occupational noise exposure in the operating area of two microenterprises in the city of Neiva was evaluated by dosimetry and sonometry. These companies are dedicated to the commercialization of chipboard using specialized machines such as vertical saws and veneers.

Methodology: Initially, a diagnosis of working conditions was made; later, noise measurements were carried out following the workbased strategy pursuant ISO 9612-2010.

Results and conclusions: The equivalent sound pressure levels (73,0 and 82,4 dB (A)) registered by the sonometry system are similar to those reported in carpentry workshops and furniture factories. The dosimetry results reveal that the vertical saw and the veneer machine operators are exposed 8 hours daily to noise levels between 88,50 and $89,9 \mathrm{~dB}(\mathrm{~A})$, which exceed the permissible limit value ( $85 \mathrm{~dB}$ ), as stipulated by resolution 1792 of 1990 . However, the chipboard microenterprise operators evaluated were not overexposed to noise thanks to the attenuation brought by the hearing protectors used.

Keywords: Industrial hygiene; noise pollution; occupational health; wood industry 


\section{RESUMEN GRÁFICO}

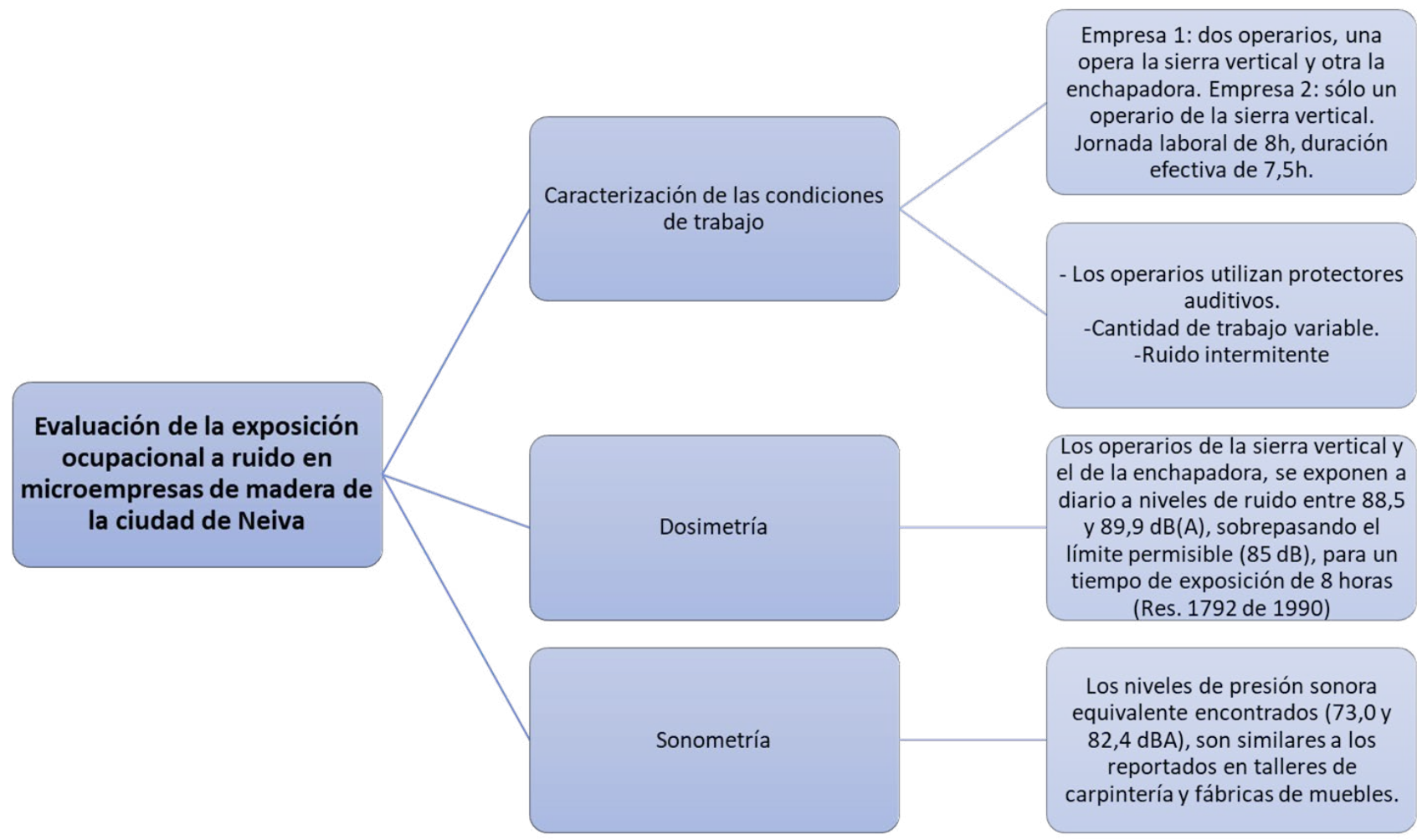

\section{INTRODUCCIÓN}

El ruido en el ambiente de trabajo se genera comúnmente por la operación de máquinas y herramientas. Debido a la dureza del material que procesan, las empresas del sector maderero emplean máquinas de trabajo pesado para aserrar, cortar, picar y arreglar la madera, que son fuentes de ruido. Se ha encontrado que los niveles de ruido producidos por las sierras de aserradero en operación varían de 80 hasta $120 \mathrm{~dB}$ (Owoyemi et al., 2017). La normatividad colombiana, en la Resolución 1792 de 1990, se adhiere a los criterios del Instituto Nacional para la Seguridad y Salud Ocupacional (NIOSH) y de la Conferencia Americana de Higienistas Industriales Gubernamentales (ACGIH), al admitir un máximo de $85 \mathrm{~dB}$ para una jornada de trabajo de 8 horas, ya que, a niveles superiores a este, se incrementa la posibilidad de pérdida de la audición. Sin embargo, la norma colombiana admite intervalos de variación de ruido cada $5 \mathrm{~dB}$, que es menos protectora del trabajador que la variación de 3 dB (Ministerio de la Protección Social, 2006).

Debido a la exposición a elevados niveles de ruido, los trabajadores del sector industrial son susceptibles a los efectos asociados a este factor, como el estrés, irritabilidad, trastornos del sueño, incremento de la presión arterial, pérdida de la capacidad auditiva, entre otros (Ganime et al., 2010). A nivel mundial se estima que el $16 \%$ de la pérdida auditiva incapacitante en adultos se atribuye al ruido ocupacional, pero este porcentaje puede variar entre el 7 y el $21 \%$ en las distintas regiones, siendo mayor en los países en vía de desarrollo (Nelson et al., 2005). Al menos 432 millones de adultos presentan pérdida auditiva incapacitante, siendo la elevada exposición a ruido una las causas (WHO, 2019). Precisamente, las patologías auditivas, ocupan el segundo lugar entre las enfermedades laborales más 
reportadas a las ARL en Colombia, después de las lesiones musculo-esqueléticas (MinTrabajo, 2013) y el Ministerio de Salud y Protección Social han estimado que la prevalencia de pérdida de audición por exposición a ruido entre la población laboralmente activa (25 a 50 años) es de un $14 \%$. La prevalencia de hipoacusia ocupacional inducida por ruido del orden del $20 \%$ y $44 \%$ en trabajadores de la madera ha sido corroborada por autores como Sierra y Bedoya (2016) y Robinson et al. (2015), respectivamente. Además, en estudios realizados en trabajadores de diferentes industrias se ha encontrado elevada asociación entre altos niveles de exposición al ruido y riesgo de accidentes laborales y lesiones (Picard et al., 2008; Palmer et al., 2008), razones suficientes para ejercer controles sobre el ruido en el ambiente laboral.

En este sentido, las mediciones de los niveles de ruido son fundamentales para obtener un diagnóstico del grado de riesgo asociado a este factor que facilite la toma de decisiones respecto a las medidas de prevención por adoptar para el control del ruido. Ha habido una gran preocupación por el ruido industrial en el sector maderero; se han desarrollado investigaciones en Grecia (Ntalos y Papadopoulos, 2013), Arabia Saudí (Noweir et al., 2014b), Nepal (Robinson et al., 2015) y Colombia (Sierra y Bedoya, 2016), que coinciden en que, en estas industrias, los niveles de ruido llegan a superar el límite permisible de los $85 \mathrm{~dB}$. Los resultados del estudio desarrollado por Sierra y Bedoya (2016), indican que el riesgo por exposición a ruido en aserríos está entre medio y alto. No obstante, la literatura todavía no presenta estudios que aborden la exposición a ruido en empresas que laboran con madera aglomerada, que son las que más abundan en nuestro contexto. Además, autores como Acero, Jaimes y Romero (2016) refieren que el ruido es un problema de salud pública que se presenta en el país y que ha sido muy poco analizado.

Por esto, el presente estudio tuvo por objetivo evaluar la exposición ocupacional a ruido en dos microempresas de madera aglomerada de la ciudad de Neiva, en el segundo semestre del año 2019. Para esto, se caracterizaron las condiciones laborales y operativas en las empresas, se verificaron los niveles de sonido percibidos por los trabajadores del área operativa mediante dosimetría y se estimó la exposición a ruido por puesto de trabajo mediante sonometría.

\section{MATERIALES Y MÉTODOS}

Se realizó un estudio descriptivo, de corte longitudinal, en dos microempresas de madera de la ciudad de Neiva que comercializan principalmente madera aglomerada y cuyas operaciones incluyen el corte y enchapado de esos materiales.

Inicialmente se llevó a cabo la caracterización de las condiciones de la labor en cada empresa, para conocer sus instalaciones, maquinaria, horario laboral, cargos, número de trabajadores, así como el empleo de elementos de protección personal (EPP) y la existencia de sistemas de control de ruido.

En la medición de la exposición al ruido, se aplicó la estrategia de medición basada en la labor, teniendo en cuenta que era muy complicado discriminar por tareas ya que estas se desarrollaban en períodos muy variables. Se siguieron las recomendaciones de medición según la ISO 9612-2010, para la dosimetría y sonometría. Los instrumentos de medición utilizados fueron Dosímetro marca 3M, modelo The Edge 5 y Sonómetro clase 1, marca CaseIla, modelo CEL-633C, ambos con certificado de calibración vigente. 
En cuanto al plan de medición de la presión sonora, según la ISO, la duración total mínima de las mediciones con el dosímetro para grupos inferiores a 5 personas debe ser de 5,5 horas; de este modo, con el dosímetro se realizaron tres mediciones de 55 minutos cada día, por dos días consecutivos, bajo condiciones ambientales similares. Las mediciones se efectuaron en dos trabajadores de la empresa 1 y uno de la empresa 2.

A partir de las lecturas registradas con el dosímetro, se calcularon parámetros como el nivel de presión sonora continuo equivalente ponderado $A$, el nivel diario de exposición al ruido ponderado A, la dosis y el nivel de sonido percibido por el trabajador que utiliza protector auditivo.

Para calcular el nivel de presión sonora continuo equivalente ponderado $A, L p, A$, eqT, $n$, se utilizó la ecuación 1 :

$L_{p A, e q T_{e}}=10 \lg \left(\frac{1}{N} \sum_{n=1}^{N} 10^{(0,1 \times L p, A, e q T, n)}\right) d B$

(Ecuación 1)

Donde:

$L_{p, A, e q T, n}$ : es el nivel de presión sonora continuo equivalente ponderado $A$, de la medición $n$.

$\mathrm{n}$ : es el número de la medición de trabajo

$\mathrm{N}$ : el número total de las mediciones de trabajo.

A partir de la ecuación 2 se calculó el nivel diario de exposición al ruido ponderado $A, L_{E x}, 8 h^{\prime}$ de cada trabajador:

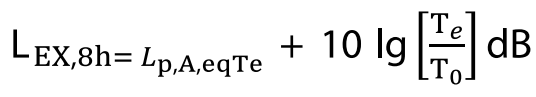

(Ecuación 2)

Donde:

$L_{p, A, e q T, e}$ : es el nivel de presión sonora continuo equivalente ponderado $\mathrm{A}$ para la duración efectiva de la jornada laboral.
$\mathrm{T}_{\mathrm{e}}$ : es la duración efectiva de la jornada laboral. $\mathrm{T}_{0}$ : es la duración de referencia, $\mathrm{T}_{0}=8 \mathrm{~h}$

La dosis promedio (D) en porcentaje, se determinó a través de la ecuación 3 :

$$
D=\left[\frac{C 1}{T 1}+\frac{C 2}{T 2}+\ldots \frac{C_{n}}{T_{n}}\right] \times 100 \text { (Ecuación 3) }
$$

Donde:

C: Es el tiempo real de exposición para cada nivel de presión sonora (NPSI).

T: Es el tiempo máximo de exposición permitido para cada nivel de presión sonora (NPSI)

Para el cálculo del nivel de sonido percibido por el trabajador que utiliza protector auditivo, se siguió el procedimiento indicado por la NIOSH (Ecuaciones 4,5 y 6), citado en la GATIHNIR del Ministerio de protección social:

$N R R_{\text {corregido }}=N R R_{\text {protector }}-7$

(Ecuación 4)

Corrección NIOSH $=N R R_{\text {corregido }}$ $\left(N R R_{\text {corregido }} \times 0,25\right)$

(Ecuación 5)

Nivel percibido por el trabajador $=$ Nivel de exposición - Corrección NIOSH

(Ecuación 6)

Para evaluar los niveles sonoros en determinados momentos de la labor, se utilizó la sonometría. esta se realizó mediante barrido alrededor de las máquinas donde se encontraba el trabajador, tomando tres puntos diferentes. En cada punto se realizaron mediciones de 15 minutos, dos días diferentes, en condiciones ambientales similares. Con los datos se determinaron los parámetros $L p, A, e q T, n$ y $\mathrm{L}_{\mathrm{Ex}, 8 \mathrm{~h}}$. También se quiso conocer la distribución de frecuencias del ruido al que están expuestos los trabajadores de la empresa 1. 


\section{Comparación estadística de los niveles de presión sonora}

Los resultados de las mediciones se analizaron a través de medidas descriptivas como la media y la desviación estándar ( $\sigma)$. Utilizando Jamovi, se compararon los niveles de presión sonora obtenidos por empresa. Jamovi es un software estadístico libre de fácil manejo que cuenta con módulos de estadística robusta, mediación y moderación, cálculo de poder estadístico, análisis factorial confirmatorio, entre otros (Sánchez Villena, 2019).

Para el análisis se conformaron dos grupos, el grupo 1 correspondía a los niveles de presión sonora obtenidos en la empresa 1 y el grupo 2 a los niveles de presión registrados en la empresa 2. Las hipótesis fueron:

HO: ambos grupos presentan medias iguales y no tienen diferencias significativas.

$\mathrm{H} 1$ : ambos grupos presentan medias diferentes y tienen diferencias significativas.

La evaluación de la hipótesis nula (H0) se realizó por medio del test paramétrico de Welch's que es una alternativa al Student's cuando las varianzas de ambos grupos son distintas. Se utiliza para comparar las medias de una variable cuantitativa continua entre dos grupos independientes determinando si existen diferencias significativas entre estas (Laguna, 2014). Pertinente en este caso donde los niveles de presión sonora constituyen la variable cuantitativa dependiente y las empresas 1 y 2 la variable cualitativa dicotómica.

\section{RESULTADOS Y DISCUSIÓN}

\section{Caracterización de las condiciones de trabajo}

Las empresas donde se realizaron las mediciones de ruido cuentan con área de ventas y área operativa dispuestas consecutivamente.
Sin embargo, en la empresa 1 , el área operativa está separada del área de ventas por un muro de ladrillo. Por otra parte, en la empresa 2, solo hay una separación parcial con una lámina de madera y vidrio, dichas separaciones constituyen un control de ingeniería de ruido. Se evidenció que, en el área de operación, en la empresa 1 , hay dos trabajadores permanentes que laboran ocho horas diarias de lunes a sábado (medio día), uno maneja la sierra vertical (marca STRIEBIG); responsable del corte de material y el otro la enchapadora (marca BRANDT); una máquina que da acabado a la madera aglomerada fijando una lámina blanca. Por otro lado, en la empresa 2, solo hay un trabajador que opera la sierra vertical de la misma marca.

La jornada laboral de ese personal comprende actividades como la recepción y organización de materiales, planificación del trabajo, el corte o enchapado de materiales y dos descansos al día de quince minutos. Como su trabajo empieza aproximadamente media hora después de encender las máquinas, se consideró una duración efectiva de la jornada laboral de 7,5 h.

El ruido en esos ambientes laborales es intermitente debido a que la actividad de corte o enchapado de madera aglomerada es variable, depende de la cantidad de trabajo que resulta en el día. Se observó que los trabajadores utilizan elementos de protección auditiva. En la empresa 1, cuentan con protectores tipo copa FONO SAMURAI STEELPRO SNR 27 DB ISP, de la marca Steelpro Safety, que tienen un nivel de reducción de ruido (NRR) de $23 \mathrm{~dB}$. Por otra parte, el trabajador de la empresa 2 utiliza un protector FONO PELTOR H10/OPTIME 105, tipo copa de la marca 3M, cuyo NRR es de $30 \mathrm{~dB}$. Ambos protectores son certificados bajo la norma ANSI S3.19. 


\section{Niveles de ruido ocupacional}

Al evaluar la exposición personal a ruido según la labor, se comprobó que los operarios de la sierra vertical (Tabla 1 ) reciben aproximadamente un nivel diario de exposición al ruido ponderado $A, L_{E x} 8 n$, de $89,1 \mathrm{~dB}$ y $89,9 \mathrm{~dB}$, con una incertidumbre expandida $\left(\mathrm{UL}_{\mathrm{Ex}, 8 \mathrm{~h}}\right)$ asociada de 3,0 dB y 3,3 dB respectivamente, para una probabilidad de cobertura unilateral del $95 \%(k=1,65)$. Por otra parte, el operario de la enchapadora se somete diariamente a un nivel de ruido ponderado $A$ de $88,50 \mathrm{~dB}$, con una incertidumbre expandida asociada de 3,3 dB. Esos niveles de ruido superan el valor límite permisible de $85 \mathrm{~dB}$ para una jornada de 8 horas, dispuesto por la resolución 1792 de 1990. De acuerdo con la misma resolución, para los niveles sonoros hallados, corresponden tiempos máximos de exposición de 4 a 5 horas, tomando como referencia una exposición al $50 \%$ de la dosis ( $80 \mathrm{~dB}$ ). Además, conforme la GATI-HNIR, los trabajadores de esas empresas se clasifican en "Muy alta exposición", por estar expuestos a dosis superiores a 85 $\mathrm{dB}(\mathrm{A})$, lo cual requiere que se sometan a evaluaciones médicas continuas.

No obstante, gracias al empleo continuo de protectores auditivos, los niveles de ruido percibidos por dichos trabajadores son menores de $85 \mathrm{~dB}$, como se observa en la tabla 1, garantizando la exposición a dosis inferiores a $50 \%$. Sin embargo, el control de ruido en el trabajador debe ser la última medida a adoptar, primero deben considerarse los controles en la fuente y en el medio (NIOSH, 1998).

Tabla 1. Niveles de ruido percibido por los operarios

\begin{tabular}{|c|c|c|c|c|c|c|c|c|c|c|}
\hline & \multirow[b]{2}{*}{ Máquina } & \multicolumn{3}{|c|}{$\begin{array}{l}\text { Niveles de presión } \\
\text { sonora (dBA) }\end{array}$} & \multirow[b]{2}{*}{ Media } & \multirow[b]{2}{*}{$\boldsymbol{\sigma}$} & \multirow[b]{2}{*}{$\begin{array}{c}\text { Nivel de } \\
\text { presión } \\
\text { sonora } \\
\text { equivalente } \\
(L p, A, \text { eqT })\end{array}$} & \multirow[b]{2}{*}{$\begin{array}{c}\text { Nivel de } \\
\text { exposición } \\
\text { de ruido } \\
\text { diario } \\
\text { ponderado } \\
\text { A (LEX, 8h) }\end{array}$} & \multirow[b]{2}{*}{$\begin{array}{l}\text { Dosis } \\
\text { (sin } \\
\text { protección) }\end{array}$} & \multirow[b]{2}{*}{$\begin{array}{c}\text { Nivel } \\
\text { de ruido } \\
\text { percibido }\end{array}$} \\
\hline & & $\begin{array}{l}L p, A, \\
\text { eqT, } 1\end{array}$ & $\begin{array}{l}L p, A, \\
\text { eqT, } 2\end{array}$ & $\begin{array}{l}L p, A, \\
\text { eqT, } 3\end{array}$ & & & & & & \\
\hline \multirow{2}{*}{$\begin{array}{l}\text { Empresa } \\
1\end{array}$} & \multirow{2}{*}{ Sierra vertical } & 89,6 & 89,3 & 88,9 & 89,3 & 0,4 & \multirow{2}{*}{$89,3 \mathrm{~dB}$} & \multirow{2}{*}{$89,1 \mathrm{~dB}$} & \multirow{2}{*}{$171 \%$} & \multirow{2}{*}{$77,1 \mathrm{~dB}$} \\
\hline & & 89,1 & 89,2 & 89,9 & 89,4 & 0,4 & & & & \\
\hline \multirow{2}{*}{$\begin{array}{l}\text { Empresa } \\
2\end{array}$} & \multirow{2}{*}{ Sierra vertical } & 86,9 & 89,9 & 88,9 & 88,6 & 1,5 & \multirow{2}{*}{$90,2 \mathrm{~dB}$} & \multirow{2}{*}{$89,9 \mathrm{~dB}$} & \multirow{2}{*}{$194 \%$} & \multirow{2}{*}{$72,7 \mathrm{~dB}$} \\
\hline & & 90,6 & 91,8 & 91,5 & 91,3 & 0,6 & & & & \\
\hline \multirow{2}{*}{$\begin{array}{l}\text { Empresa } \\
1\end{array}$} & \multirow[b]{2}{*}{ Enchapadora } & 87 & 88,1 & 89,3 & 88,1 & 1,2 & \multirow[b]{2}{*}{$88,79 \mathrm{~dB}$} & \multirow[b]{2}{*}{88,5} & \multirow[b]{2}{*}{$158 \%$} & \multirow[b]{2}{*}{$76,5 \mathrm{~dB}$} \\
\hline & & 86,8 & 90,2 & 90,1 & 89,0 & 1,9 & & & & \\
\hline
\end{tabular}

Fuente: Autores

Los resultados de la evaluación sonora por puesto de trabajo en el área operativa (Tabla 2), exhiben que los niveles de ruido diario $\left(L_{E X, 8 \mathrm{~h}}\right)$ están por debajo del límite permisible de exposición, ya que oscilan entre $73,0 \mathrm{~dB}$ y $82,4 \mathrm{~dB}$. El $\mathrm{L}_{E x, 8 \mathrm{~h}}$ en la enchapadora fue mayor que en la sierra vertical porque se presentó la condición de que ambas máquinas operaron simultáneamente.

Posiblemente, los niveles de $\mathrm{L}_{E X^{\prime}}$ 8h' obtenidos a partir de las mediciones con el sonómetro fueron inferiores a los registrados con el dosímetro porque este último integra, en un solo 
valor (dosis), la magnitud de la energía recibida en un período de tiempo, que es un acumulado de la exposición. En contraste, el sonómetro mide el nivel de sonido en un solo punto en el tiempo, proporciona una instantánea del sonido en un momento determinado, siendo útil cuando el sonido es estable con poca variación (Johnson, s. f.). Por esta razón la GATI-HNIR sugiere el empleo de dosímetros para la medición de ruido en ambientes de trabajo donde los niveles de sonido fluctúan.

Tabla 2. Niveles de sonido percibidos en el puesto de trabajo

\begin{tabular}{|c|c|c|c|c|c|c|c|c|}
\hline & \multirow[b]{2}{*}{ Máquina } & \multicolumn{3}{|c|}{$\begin{array}{c}\text { Niveles de presión } \\
\text { sonora (dBA) }\end{array}$} & \multirow[b]{2}{*}{ Media } & \multirow[b]{2}{*}{$\boldsymbol{\sigma}$} & \multirow[b]{2}{*}{$\begin{array}{c}\text { Nivel de } \\
\text { presión sonora } \\
\text { equivalente } \\
(L p, A, \text { eqT })\end{array}$} & \multirow[b]{2}{*}{$\begin{array}{l}\text { Nivel de exposición } \\
\text { de ruido diario } \\
\text { ponderado A } \\
\text { (LEX, 8h) }\end{array}$} \\
\hline & & $\begin{array}{l}L p, A, \\
\text { eqT,1 }\end{array}$ & $\begin{array}{l}L p, A, \\
\text { eqT, } 2\end{array}$ & $\begin{array}{l}L p, A, \\
\text { eqT, } 3\end{array}$ & & & & \\
\hline \multirow{2}{*}{ Empresa 1} & \multirow{2}{*}{ Sierra vertical } & 70,5 & 69,8 & 74,2 & 71,5 & 2,4 & \multirow{2}{*}{$79,0 \mathrm{~dB}$} & \multirow{2}{*}{$73,3 \mathrm{~dB}$} \\
\hline & & 71,6 & 79,8 & 85,1 & 78,8 & 6,8 & & \\
\hline \multirow{2}{*}{ Empresa 2} & \multirow{2}{*}{ Sierra vertical } & 71,5 & 70,4 & 75,7 & 72,5 & 2,8 & \multirow{2}{*}{$78,8 \mathrm{~dB}$} & \multirow{2}{*}{$73,0 \mathrm{~dB}$} \\
\hline & & 68,4 & 70,2 & 76,8 & 71,8 & 4,4 & & \\
\hline \multirow{2}{*}{ Empresa 1} & \multirow{2}{*}{ Enchapadora } & $53,5^{*}$ & 72,3 & 84,8 & 70,2 & 15,8 & \multirow{2}{*}{$82,7 \mathrm{~dB}$} & \multirow{2}{*}{$82,4 \mathrm{~dB}$} \\
\hline & & 83,5 & 84,7 & 84,5 & 84,2 & 0,6 & & \\
\hline
\end{tabular}

Fuente: Autores

En estudios de evaluación de ruido en entornos laborales de talleres de carpintería y empresas de fornitura (Noweir et al., 2014; Robinson et al., 2015) se hallaron niveles de $\boldsymbol{L} \boldsymbol{p}, \boldsymbol{A}, \mathbf{e q T}$, en el rango de 77,9 y 79,4 dB (A) próximos a los encontrados en el presente estudio.

La variación de los niveles sonoros de un día para otro y entre empresas de actividades semejantes, se deben a las múltiples variables que intervienen en la generación de ruido. Además de las características del material, cantidades y espesor de la pieza trabajada, los niveles de ruido dependen también del tipo y ajuste de la máquina, velocidad de corte, número de trabajadores y máquinas involucradas, otra variable es el sistema de extracción de viruta, donde el ruido varía en función de la velocidad y el diseño del sistema (NTP-ISO 9612, 2010; Instituto Andaluz de Prevención de Riesgos Laborales, s. f.).

\section{Comparación estadística de los niveles de presión sonora}

Los niveles de presión sonora presentados en las Tablas 1 y 2 se compararon entre empresas mediante la hipótesis nula (HO: ambos grupos presentan medias iguales y no tienen diferencias significativas) empleando el test de Welch's a un nivel de significancia (a) de 0,05 como alternativa al Student's, debido a que las varianzas de los grupos fueron diferentes (Jamovi, 2019).

Como se observa en la Tabla 3, dado que $\mathrm{p}>$ 0,05 se acepta la hipótesis nula y por lo tanto 
los estadísticos de los grupos que se compararon, en este caso los niveles de presión sonora no fueron significativamente diferentes entre las empresas evaluadas con una probabilidad mayor del 95\%. Otros estudios en la industria maderera (Noweir et al., 2014; Sierra Calderón y Bedoya Marrugo, 2016) reportaron variaciones de los niveles de presión sonora entre empresas similares sin embargo no evaluaron estadísticamente dicha diferencia.

Tabla 3. Resultado prueba de hipótesis

\begin{tabular}{llc} 
Variable dependiente & $\begin{array}{l}\text { Variable } \\
\text { de grupo }\end{array}$ & $\mathbf{p}$ \\
$\begin{array}{l}\text { Niveles de presión } \\
\text { sonora }(\mathrm{n}=36)\end{array}$ & Empresas & 0,387 \\
\hline
\end{tabular}

Fuente: Autores

\section{CONCLUSIONES}

En las condiciones operativas evaluadas en el presente estudio, se corroboró que los trabajadores del área operativa de empresas que comercializan madera aglomerada están expuestos a niveles de ruido diario $\left(L_{E x, 8 h}\right)$ que superan el límite permisible de $85 \mathrm{~dB}$ para una jornada laboral de $8 \mathrm{~h}$, como precisa la resolución 1792 de 1990. A pesar de esto, el uso de protectores auditivos garantiza que esos trabajadores no se encuentren sobreexpuestos a este factor.

Este es uno de los primeros estudios que aborda el ruido ocupacional en microempresas de madera aglomerada donde se emplean máquinas modernas como sierras verticales y enchapadoras. Los niveles de presión sonora equivalente ( $\boldsymbol{L p}, \boldsymbol{A}, \boldsymbol{e q T})$ hallados en este estudio se aproximan a los encontrados en algunos talleres de carpintería (Noweir et al., 2014) y fábricas de muebles (Robinson et al., 2015); sin embargo, resultan inferiores cuando se comparan con los $\boldsymbol{L p}, \boldsymbol{A}, \mathbf{e q T}$ reportados en aserríos (Kolodziej y Cruz, 2013; Pozo, 2010;
Sierra y Bedoya, 2016) también empleando sonometría. Probablemente debido a que las maquinas que utilizan en esas empresas son más rústicas y antiguas.

Como en este estudio fueron pocas las empresas evaluadas y las mediciones limitadas, se recomienda ampliar la muestra de estudio y adoptar una estrategia de medición de jornada completa para corroborar la diferencia entre los resultados que proporcionan los instrumentos de medición de ruido. Asimismo, se recomienda un análisis sonoro de las máquinas para conocer los principales focos generadores de ruido para actuar sobre estos.

\section{CONTRIBUCIÓN DE LA AUTORÍA}

Ingrith M. Romero Méndez: metodología, investigación, análisis de datos, conceptualización, escritura - revisión y edición. Dinory Serrato: asesoría técnica y recolección de datos. Roberson Daniel Bernal Medina: recolección de datos y procesamiento de los mismos. Jhonathan Cabrera Urriago: gestión de equipos de medición, recolección de datos.

\section{AGRADECIMIENTOS}

Agradecimiento especial al equipo del semillero JIASSO del Programa de Administración de la Salud Ocupacional de Neiva, que colaboró en la ejecución de la investigación. A la profesora Dinory Serrato, Roberson Bernal, Jhonathan Cabrera y Juan Carlos Morales. El presente artículo se deriva del trabajo desarrollado por Bernal R. y Cabrera J. (2019) y dirigido por la autora.

\section{LITERATURA CITADA}

Acero, J., Jaimes, M., y Romero, G. A. (2016). Generación de mapas de ruido (industrial) desde sistemas de información geográfica. Un acercamiento desde la literatura. Revista Tecnura, 20 (49), 152. doi: 10.14483/udistrital.jour. tecnura.2016.3.a10 
Bernal R. y Cabrera J. (2019). Evaluación del riesgo asociado al ruido en trabajadores de empresas comercializadoras de madera aglomerada de la ciudad de Neiva (tesis de pregrado). Corporación Universitaria Minuto de Dios, Neiva, Colombia.

Ganime, J. F., Almeida da Silva, L., Robazzi, M. do C. C., Valenzuela, S., y Faleiro, S. A. (2010). El ruido como riesgo laboral: Una revisión de la literatura. Enfermería Global, (19). doi:10.4321/ S1695-61412010000200020

Instituto Andaluz de Prevención de Riesgos Laborales. (2013). Guía de buenas prácticas contra el ruido en la industria de la madera.pdf. Recuperado de https://www.juntadeandalucia.es/export/ drupaljda/Gu\%C3\%ADa\%20de\%20buenas\%20 practicas $\% 20$ contra $\% 20$ el $\% 20$ ruido $\% 20$ en $\% 20$ la\%20industria\%20de\%20la\%20madera.pdf

Johnson, P. T. (s. f.). Noise Exposure: Explanation of OSHA and NIOSH Safe-Exposure Limits and the Importance of Noise Dosimetry. Recuperado de https://www.etymotic.com/downloads/dl/ file/id/47/product/307/noise_exposure_explanation_of_osha_and_niosh_safe_exposure_limits_and_the_importance_of_noise_dosimetry.pdf

Kolodziej, F.S y Cruz R. E. (2013). Exposición al Ruido Ambiental y Laboral de los Trabajadores de una Industria Maderera. Argentina: Estrucplan. Recuperado de https://estrucplan.com.ar/ exposicion-al-ruido-ambiental-y-laboral-de-lostrabajadores-de-una-industria-maderera/

Laguna C. (2014). Inferencia paramétrica: relación entre variables cualitativas y cuantitativas. Recuperado de http://www.ics-aragon.com/cursos/ salud-publica/2014/pdf/M2T08.pdf

Ministerio del Trabajo (2013). Segunda Encuesta Nacional de Condiciones de Seguridad y Salud en el Trabajo en el Sistema General de Riesgos Laborales. Recuperado de https://fasecolda.com/ cms/wp-content/uploads/2019/08/ii-encuestanacional-seguridad-salud-trabajo-2013.pdf

Ministerio de la Protección Social. (2006). Guía de atención integral de salud ocupacional basada en la evidencia para Hipoacusia Neurosensorial Inducida por ruido en el trabajo, GATI-HNIR. Recuperado de: https://www.minsalud.gov.co/ Documentos\%20y\%20Publicaciones/GATISOHIPOACUSIA\%20NEROSENSORIAL.pdf
Nelson, D. I., Nelson, R. Y., Concha-Barrientos, M., y Fingerhut, M. (2005). The global burden of occupational noise-induced hearing loss. American Journal of Industrial Medicine, 48(6), 446-458. doi:10.1002/ajim.20223

NTP-ISO 9612. (2010). Acústica. Determinación de la exposición a ruido laboral. Método de ingeniería. Recuperado de https://www.academia.edu/9753456/Determinaci\%C3\%B3n de_la_exposici\%C3\%B3n_a_ruido_laboral_ ISO_9612_2010

Noweir, M. H., Bafail, A. O., y Jomoah, I. M. (2014). Noise Pollution in Metalwork and Woodwork Industries in the Kingdom of Saudi Arabia. International Journal of Occupational Safety and Ergonomics, 20(4), 661-670. doi:10.1080/1080354 8.2014.11077068

Ntalos, G. A., y Papadopoulos, A. N. (2013). Noise Emission Levels in Greek Wood and Furniture Processing Industry. Journal of the Institute of Wood Science, 17(2), 99-103. doi:10.1179/ wsc. 2005.17.2.99

Owoyemi, M. J., Falemara, B., y Owoyemi, A. J. (2017). Noise Pollution and Control in Wood Mechanical Processing Wood Industries. Biomedical Statistics and Informatics, 2(2): 54-60. doi: 10.11648/j.bsi.20170202.13

Palmer, K. T., Harris, E. C., y Coggon, D. (2008). Chronic health problems and risk of accidental injury in the workplace: A systematic literature review. Occupational and Environmental Medicine, 65(11), 757-764. doi:10.1136/oem.2007.037440

Picard, M., Girard, S. A., Simard, M., Larocque, R., Leroux, T., y Turcotte, F. (2008). Association of work-related accidents with noise exposure in the workplace and noise-induced hearing loss based on the experience of some 240,000 person-years of observation. Accident Analysis \& Prevention, 40(5), 1644-1652. doi:10.1016/j. aap.2008.05.013

Pozo, E. F. (2010). Estudio de Ruido Generado en la Industria Maderera en la Ciudad de Cuenca y sus efectos a la Salud (Tesis de maestría). Universidad de Cuenca, Cuenca, Ecuador. Recuperado de http://dspace.ucuenca.edu.ec/bitstream/123456789/2585/1/tm4335.pdf

Robinson, T., Whittaker, J., Acharya, A., Singh, D., y Smith, M. (2015). Prevalence of noise-induced 
hearing loss among woodworkers in Nepal: A pilot study. International Journal of Occupational and Environmental Health, 21(1), 14-22. doi:/10. 1179/2049396714Y.0000000084

Sánchez Villena, A. (2019). Uso de programas estadísticos libres para el análisis de datos: Jamovi, Jasp y R. Revista Perspectiva, 20(1), 112-114. doi:10.33198/rp.v20i1.00026
Sierra Calderón, D. D., y Bedoya Marrugo, E. A. (2016). Prevalencia de hipoacusia neurosensorial inducida por ruido en empresas del sector madera de la ciudad de Cartagena. 2015. Nova, 14(25), 47. doi:10.22490/24629448.1726

WHO. (2019). Deafness and Hearing Loss. World Health Organization. Recuperado de https:// www.who.int/news-room/fact-sheets/detail/deafness-and-hearing-loss

\section{(9) $(1) \Theta(9$}


